\title{
Some New Tempered Fractional Pólya-Szegö and Chebyshev-Type Inequalities with Respect to Another Function
}

\author{
Gauhar Rahman $\mathbb{D}^{1},{ }^{1}$ Kottakkaran Sooppy Nisar, ${ }^{2}$ Thabet Abdeljawad $\mathbb{D}^{3,4,5}$ \\ and Muhammad Samraiz ${ }^{6}$ \\ ${ }^{1}$ Department of Mathematics, Shaheed Benazir Bhutto University, Sheringal 18000, Upper Dir, Pakistan \\ ${ }^{2}$ Department of Mathematics, College of Arts and Sciences, Prince Sattam Bin Abdulaziz University, \\ Wadi Aldawaser 11991, Saudi Arabia \\ ${ }^{3}$ Department of Mathematics and General Sciences, Prince Sultan University, Riyadh, KSA, Saudi Arabia \\ ${ }^{4}$ Department of Medical Research, China Medical University, Taichung 40402, Taiwan \\ ${ }^{5}$ Department of Computer Science and Information Engineering, Asia University, Taichung 40402, Taiwan \\ ${ }^{6}$ Department of Mathematics, University of Sargodha, Sargodha, Pakistan
}

Correspondence should be addressed to Thabet Abdeljawad; tabdeljawad@psu.edu.sa

Received 18 April 2020; Accepted 3 August 2020; Published 2 November 2020

Academic Editor: Tepper L Gill

Copyright ( $) 2020$ Gauhar Rahman et al. This is an open access article distributed under the Creative Commons Attribution License, which permits unrestricted use, distribution, and reproduction in any medium, provided the original work is properly cited.

\begin{abstract}
In this present article, we establish certain new Pólya-Szegö-type tempered fractional integral inequalities by considering the generalized tempered fractional integral concerning another function $\Psi$ in the kernel. We then prove certain new Chebyshev-type tempered fractional integral inequalities for the said operator with the help of newly established Pólya-Szegö-type tempered fractional integral inequalities. Also, some new particular cases in the sense of classical tempered fractional integrals are discussed. Additionally, examples of constructing bounded functions are considered. Furthermore, one can easily form new inequalities for Katugampola fractional integrals, generalized Riemann-Liouville fractional integral concerning another function $\Psi$ in the kernel, and generalized fractional conformable integral by applying different conditions.
\end{abstract}

\section{Introduction}

The well-known Chebyshev functional [1] is defined by

$$
\mathscr{T}\left(f_{1}, f_{2}\right)=\frac{1}{x_{2}-x_{1}} \int_{x_{1}}^{x_{2}} f_{1}(\theta) f_{2}(\theta) \mathrm{d} \theta-\frac{1}{x_{2}-x_{1}}\left(\int_{x_{1}}^{x_{2}} f_{1}(\theta) \mathrm{d} \theta\right) \frac{1}{x_{2}-x_{1}}\left(\int_{x_{1}}^{x_{2}} f_{2}(\theta) \mathrm{d} \theta\right)
$$

where the functions $f_{1}$ and $f_{2}$ are integrable on $\left[x_{1}, x_{2}\right]$. If the functions $f_{1}$ and $f_{2}$ are synchronous on $\left[x_{1}, x_{2}\right]$, i.e.,

$$
\left(f_{1}(\vartheta)-f_{1}(\zeta)\right)\left(f_{2}(9)-f_{2}(\zeta)\right) \geq 0,
$$

for any $\vartheta, \zeta \in\left[x_{1}, x_{2}\right]$, then $\mathscr{T}\left(f_{1}, f_{2}\right) \geq 0$. Functional (1) has gained more recognition due to its diverse applications in the fields of transform theory, numerical quadrature, probability, and statistical problems. Additionally, the researchers have established a large number of integral 
inequalities by utilizing functional (1). The interesting readers may consult [2-5]. In [6], Tassaddiq et al. recently established certain inequalities via fractional conformable integrals by considering functional (1).

In [7], Grüss introduced the following inequality:

$$
\left|\mathscr{T}\left(f_{1}, f_{2}\right)\right| \leq \frac{\left(M_{1}-m_{1}\right)\left(N_{1}-n_{1}\right)}{4},
$$

where the functions $f_{1}$ and $f_{2}$ are integrable on $\left[x_{1}, x_{2}\right]$ such that $f_{1}$ and $f_{2}$ satisfy the inequalities $m_{1} \leq f_{1}(\vartheta) \leq M_{1}$ and $n_{1} \leq f_{2}(\zeta) \leq N_{1}$, for all $\vartheta, \zeta \in\left[x_{1}, x_{2}\right]$ and for some constant $m_{1}, n_{1}, M_{1}, N_{1} \in \mathbb{R}$.

In [8], Pólya-Szegö presented the following inequality:

$$
\frac{\int_{x_{1}}^{x_{2}} f_{1}^{2}(\theta) \mathrm{d} \theta \int_{x_{1}}^{x_{2}} f_{2}^{2}(\theta) \mathrm{d} \theta}{\left(\int_{x_{1}}^{x_{2}} f_{1}(\theta) f_{2}(\theta) \mathrm{d} \theta\right)^{2}} \leq \frac{1}{4}\left(\sqrt{\frac{M_{1} N_{1}}{m_{1} n_{1}}}+\sqrt{\frac{m_{1} n_{1}}{M_{1} N_{1}}}\right)^{2} .
$$

In [9], Dragomir and Diamond presented the following inequality with the help of Pólya-Szegö inequality:

$$
\begin{aligned}
\left|\mathscr{T}\left(f_{1}, f_{2}\right)\right| \leq & \frac{\left(M_{1}-m_{1}\right)\left(N_{1}-n_{1}\right)}{4\left(x_{2}-x_{1}\right)^{2} \sqrt{m_{1} M_{1} n_{1} N_{1}}} \int_{x_{1}}^{x_{2}} f_{1}(\theta) \mathrm{d} \theta \\
& \cdot \int_{x_{1}}^{x_{2}} f_{2}(\theta) \mathrm{d} \theta,
\end{aligned}
$$

where the functions $f_{1}$ and $f_{2}$ are positive and integrable on $\left[x_{1}, x_{2}\right]$ such that $f_{1}$ and $f_{2}$ satisfy the inequalities $m_{1} \leq f_{1}(\theta) \leq M_{1}$ and $n_{1} \leq f_{2}(\zeta) \leq N_{1}$, for all $\theta, \zeta \in\left[x_{1}, x_{2}\right]$ and for some constant $m_{1}, n_{1}, M_{1}, N_{1} \in \mathbb{R}$.

In the last few decades, the researchers have considered that fractional integral inequalities are the most powerful tools for the development of both applied and pure mathematics. In [10], the authors presented some Grüss-type integral inequalities by considering fractional integrals. Some new integral inequalities in sense of Riemann-Liouville fractional integrals can be found in the work of Dahmani [11].

In [12], Sarikaya et al. gave the idea of generalized $(k, s)$-fractional integrals with applications. Set et al. [13] investigated some Grüss-type inequalities by considering generalized $k$-fractional integrals.

Very recently, the idea of fractional conformable and proportional fractional integral operators was proposed by Jarad et al. [14, 15]. Later on, Huang et al. [16] presented generalized Hermite-Hadamard-type inequalities by considering generalized fractional conformable integrals. In [17], Qi et al. established Chebyshev-type inequalities for generalized fractional conformable integrals.

In [18], Ntouyas et al. investigated some new Pólya-Szegö- and Chebyshev-type inequalities by considering Riemann-Liouville fractional integrals. The tempered fractional integral was first studied by Buschman [19], but Li et al. [20] and Meerschaert et al. [21] have described the associated tempered fractional calculus more explicitly. Fernandez and Ustaoğlu [22] investigated several analytic properties of the tempered fractional integral. In [23], Fahad et al. proposed the general form of the generalized tempered fractional integral concerning another function. In this paper, we investigate the said inequalities for the so-called tempered fractional integrals containing another function in the kernel.

The structure of the paper as follows.

In Section 2, some basic definitions are presented. Some new Pólya-Szegö-type for the so-called generalized tempered fractional integral in the sense of another function is presented in Section 3. In Section 4, we present some new generalized Chebyshev-type tempered fractional integral inequalities. In Section 5, certain new particular cases in terms of classical tempered fractional integrals are discussed. An example of constructing bounding functions is considered in Section 6. Finally, the concluding remarks are discussed in Section 7.

\section{Preliminaries}

In this section, we consider some well-known definitions and mathematical preliminaries.

Definition 1 (see [7]). Suppose that the functions $f_{1}, f_{2}:\left[x_{1}, x_{1}\right] \longrightarrow \mathbb{R}$ are positive with $\mathscr{A} \leq f_{1}(\vartheta) \leq \mathscr{B}$ and $\mathscr{C} \leq f_{2}(\vartheta) \leq \mathscr{D}$, for all $\vartheta \in\left[x_{1}, x_{1}\right]$, then the following inequality holds:

$$
\left|\frac{1}{x_{2}-x_{1}} \int_{x_{1}}^{x_{2}} f_{1}(\vartheta) f_{2}(\vartheta) \mathrm{d} \vartheta-\frac{1}{x_{2}-x_{1}} \int_{x_{1}}^{x_{2}} f_{1}(\vartheta) \mathrm{d} \vartheta \frac{1}{x_{2}-x_{1}} \int_{x_{1}}^{x_{2}} f_{2}(\vartheta) \mathrm{d} \vartheta\right| \leq \frac{1}{4}(\mathscr{B}-\mathscr{A})(\mathscr{D}-\mathscr{C}),
$$

where the constants $\mathscr{B}, \mathscr{A}, \mathscr{C}, \mathscr{D} \in \mathbb{R}$ and $1 / 4$ is the sharp of inequality (6).
Definition 2 (see $[24,25]$ ). The function $f_{1}$ will be in the space $\left.L_{p, r}\right] 0, \infty[$ if

$$
L_{p, r}\left[0, \infty\left[=\left\{f_{1}:\left\|f_{1}\right\|_{L_{p, r}[0, \infty[}=\left(\int_{r}^{s}\left|f_{1}(\vartheta)\right|^{p} \vartheta^{r} \mathrm{~d} \vartheta\right)^{1 / p}<\infty, 1 \leq p<\infty, r \geq 0\right\} .\right.\right.
$$


If we apply $r=0$, then (7) gives

$$
L_{p}\left[0, \infty\left[=\left\{f_{1}:\left\|f_{1}\right\|_{L_{p}[0, \infty[}=\left(\int_{r}^{s}\left|f_{1}(\vartheta)\right|^{p} \mathrm{~d} \vartheta\right)^{1 / p}<\infty, 1 \leq p<\infty\right\} .\right.\right.
$$

Definition 3 (see [26]). Suppose that the function $f_{1} \in L_{1}[0, \infty$ [ and assume that the function $\Psi$ is positive, monotone, and increasing on $[0, \infty[$ and having continuous derivative $\Psi^{\prime}$ on $[0, \infty[$ with $\Psi(0)=0$. Then, the Lebesgue real-valued measurable function $f_{1}$ defined on $[0, \infty$ [ is said to be in the space $X_{\Psi}^{p}(0, \infty),(1 \leq p<\infty)$ if

$$
\left\|f_{1}\right\|_{X_{\Psi}^{p}}=\left(\int_{r}^{s}\left|f_{1}(\vartheta)\right|^{p} \Psi^{\prime}(\vartheta) \mathrm{d} \vartheta\right)^{1 / p}<\infty, 1 \leq p<\infty .
$$

When $p=\infty$, then

$$
\left\|f_{1}\right\|_{X_{\Psi}^{\infty}}=\text { ess } \sup _{0 \leq \vartheta<\infty}\left[\Psi^{\prime}(\vartheta) f_{1}(\varrho)\right] .
$$

Note that, the space $X_{\Psi}^{p}(0, \infty)$ coincides with the space $L_{p}[0, \infty[$ if $\Psi(\vartheta)=\vartheta$ for $1 \leq p<\infty$ and similarly with the space $L_{p, r}[1, \infty[$ if $\Psi(\vartheta)=\ln \vartheta$ for $1 \leq p<\infty$.

Definition 4 (see [20-22]). The left-sided tempered fractional integral of order $\kappa>0$ and $\tau \geq 0$ with $\mathfrak{R}(\kappa)>0$ and $\mathfrak{R}(\tau) \geq 0$ is defined by

$$
\left({ }_{x_{1}} \mathscr{R}^{\kappa, \tau} f_{1}\right)(\theta)=\frac{1}{\Gamma(\kappa)} \int_{x_{1}}^{\theta} e^{-\tau(\theta-\vartheta)}(\varrho-t)^{\kappa-1} f_{1}(\vartheta) \mathrm{d} \vartheta, x_{1}<\theta .
$$

Remark 1. By setting $\tau=0$ in (11) yields the following Riemann-Liouville fractional integral, which is defined by

$$
\left({ }_{x_{1}} \mathscr{R}^{\kappa} f_{1}\right)(\theta)=\frac{1}{\Gamma(\kappa)} \int_{x_{1}}^{\theta}(\theta-\vartheta)^{\kappa-1} f_{1}(\vartheta) \mathrm{d} \vartheta, x_{1}<\theta
$$

The following results for (11) hold:

$$
{ }_{x_{1}} \mathscr{R}_{x_{1}}^{\kappa, \tau} \mathscr{R}^{\lambda, \tau} f_{1}(\theta)={ }_{x_{1}} \mathscr{R}^{\kappa+\lambda, \tau} f_{1}(\theta) .
$$

We define the following one-sided tempered fractional integral.

Definition 5. The one-sided tempered fractional integral of order $\kappa>0, \tau \geq 0$ is defined by

$$
\left(\mathscr{R}_{0}^{\kappa, \tau} f_{1}\right)(\theta)=\left(\mathscr{R}^{\kappa, \tau} f_{1}\right)(\theta)=\frac{1}{\Gamma(\kappa)} \int_{0}^{\theta} e^{-\tau(\theta-\vartheta)}(\theta-\vartheta)^{\kappa-1} f_{1}(\vartheta) \mathrm{d} \vartheta .
$$

Definition 6 (see [23]). Let the function $f_{1}$ be an integrable in the space $X_{\Psi}^{p}(0, \infty)$ and assume that the function $\Psi$ is positive, monotone, and increasing on $[0, \infty[$, and its derivative $\Psi^{\prime}$ is continuous on $[0, \infty[$ with $\Psi(0)=0$. Then, the left-sided generalized tempered fractional integral of the function $f_{1}$ concerning another function $\Psi$ in the kernel is defined by

$$
\left({ }_{x_{1}}^{\Psi} \mathscr{R}^{\kappa, \tau} f_{1}\right)(\theta)=\frac{1}{\Gamma(\kappa)} \int_{x_{1}}^{\theta} e^{-\tau(\Psi(\theta)-\Psi(\theta))}(\Psi(\theta)-\Psi(\theta))^{\kappa-1} \Psi^{\prime}(\theta) f_{1}(\theta) \mathrm{d} \vartheta, x_{1}<\theta
$$

where $\tau \geq 0, \kappa, \in \mathbb{C}$ with $\Re(\kappa)>0$, and $\Gamma($.$) is the well-known$ gamma function.

Remark 2. The following results can be obtained:

(i) Applying Definition 6 for $\Psi(\theta)=\theta$, we get (11). (ii) Applying Definition 6 for $\tau=0$, then it will reduce to the left-sided generalized Riemann-Liouville fractional integral operator [27].

(iii) Applying Definition 6 for $\Psi(\theta)=\ln \theta$, then it will reduce to the following left-sided Hadamard tempered integral defined by [23]

$$
\left({ }_{x_{1}}^{\Psi} \mathscr{R}^{\kappa, \tau} f_{1}\right)(\theta)=\frac{1}{\Gamma(\kappa)} \int_{x_{1}}^{\theta} \exp [-\tau(\ln \theta-\ln \vartheta)](\ln \theta-\ln \vartheta)^{\kappa-1} \frac{f_{1}(\vartheta)}{\vartheta} \mathrm{d} \vartheta, x_{1}<\theta
$$


(iv) Applying Definition 6 for $\Psi(\theta)=\theta^{\eta} / \eta, \eta>0$ and $\tau=0$, then it will reduce to the left-sided Katugampola [24] fractional integral.

(v) Applying Definition 6 for $\Psi(\theta)=\theta$ and $\tau=0$, the left Riemann-Liouville fractional integral (12) will be obtained

(vi) Applying Definition 6 for $\Psi(\theta)=\theta^{\alpha+s} / \alpha+s$ and $\tau=0$ (where $\alpha \in(0,1], \quad s \in \mathbb{R}$ and $\alpha+$ $s \neq 0$ ), then it reduces to the left-sided generalized fractional conformable integral given by [28] (vii) Applying Definition 6 for $\Psi(\theta)=\left(\theta-x_{1}\right)^{\alpha} / \alpha$, $\alpha>0$, then it reduces to the fractional conformable integral defined by Jarad et al. [14].

In this article, we consider the following one-sided GTFintegral.

Definition 7. Let the function $f_{1}$ be integrable in the space $X_{\Psi}^{p}(0, \infty)$ and assume that the function $\Psi$ is positive, monotone, and increasing on $\left[0, \infty\left[\right.\right.$, and its derivative $\Psi^{\prime}$ is continuous on $[0, \infty[$ with $\Psi(0)=0$. Then, the one-sided generalized tempered fractional integral of the function $f_{1}$ concerning another function $\Psi$ in the kernel is defined by

$$
\left({ }^{\Psi} \mathscr{R}_{0}^{\kappa, \tau} f_{1}\right)(\theta)=\frac{1}{\Gamma(\kappa)} \int_{0}^{\theta} e^{-\tau(\Psi(\theta)-\Psi(\theta))}(\Psi(\theta)-\Psi(\theta))^{\kappa-1} \Psi^{\prime}(\theta) f_{1}(\theta) \mathrm{d} \vartheta
$$

Definition 8. For $0=x_{0}<x_{1}<\cdots<x_{p}<x_{p+1}=X$, we define the following subintegrals for the generalized tempered integral

$$
\left({ }^{\Psi} \mathscr{R}_{x_{i}, x_{i+1}}^{\kappa, \tau} f_{1}\right)(X)=\frac{1}{\Gamma(\kappa)} \int_{x_{i}}^{x_{i+1}} e^{-\tau(\Psi(X)-\Psi(\vartheta))}(\Psi(X)-\Psi(\vartheta))^{\kappa-1} \Psi^{\prime}(\vartheta) f_{1}(\vartheta) \mathrm{d} \vartheta
$$

Note that

$$
\begin{aligned}
& \left({ }^{\Psi} \mathscr{R}_{0}^{\kappa, \tau} f_{1}\right)(X)=\sum_{i=0}^{p}{ }^{\Psi} \mathscr{R}_{t_{i}, t_{i+1}}^{\kappa, \tau}\left(f_{1}\right)(X)=\frac{1}{\Gamma(\kappa)} \int_{0}^{x_{1}} e^{-\tau(\Psi(X)-\Psi(\vartheta))}(\Psi(X)-\Psi(\vartheta))^{\kappa-1} \Psi^{\prime}(\vartheta) f_{1}(\vartheta) \mathrm{d} \vartheta \\
& +\frac{1}{\Gamma(\kappa)} \int_{x_{1}}^{x_{2}} e^{-\tau(\Psi(X)-\Psi(\vartheta))}(\Psi(X)-\Psi(\vartheta))^{\kappa-1} \Psi^{\prime}(\vartheta) f_{1}(\vartheta) \mathrm{d} \vartheta \\
& +\cdots+\frac{1}{\Gamma(\kappa)} \int_{x_{p}}^{X} e^{-\tau(\Psi(X)-\Psi(\vartheta))}(\Psi(X)-\Psi(\vartheta))^{\kappa-1} \Psi^{\prime}(\vartheta) f_{1}(\vartheta) \mathrm{d} \vartheta
\end{aligned}
$$

Remark 3. If we set $\Psi(X)=X$ and $\tau=0$, then (18) will reduce to the subintegrals of Riemann-Liouville fractional integral defined by [18].

\section{Pólya-Szegö-Type Tempered Fractional Integral Inequalities}

In this section, we provide some new Pólya-Szegö-type tempered fractional integral inequalities for positive and integrable functions via tempered fractional integral (17) containing another function $\Psi$ in the kernel.

Lemma 1. Let the functions $f_{1}$ and $f_{2}$ be positive and integrable on $[0, \infty)$ and assume that the function $\Psi$ is positive, monotone, and increasing on [0, $\left[\right.$, and its derivative $\Psi^{\prime}$ is continuous on $[0, \infty[$ with $\Psi(0)=0$. Suppose that $\mathscr{U}_{1}, \mathscr{U}_{2}, \mathscr{V}_{1}$ and $\mathscr{V}_{2}$ are four positive and integrable functions on $[0, \infty)$ such that

$$
\begin{aligned}
\left(H_{1}\right) 0 & <\mathscr{U}_{1}(\vartheta) \leq f_{1}(\vartheta) \leq \mathscr{U}_{2}(\vartheta), 0<\mathscr{V}_{1}(\vartheta) \leq f_{2}(\vartheta) \\
& \leq \mathscr{V}_{2}(\vartheta), \vartheta \in[0, \theta], \theta>0 .
\end{aligned}
$$

Then, for $\kappa>0, \tau \geq 0$, and $\theta>0$, the following tempered fractional integral inequality holds:

$$
\frac{{ }^{\Psi} \mathscr{R}_{0}^{\kappa, \tau}\left(\mathscr{V}_{1} \mathscr{V}_{2} f_{1}^{2}\right)(\theta)^{\Psi} \mathscr{R}_{0}^{\kappa, \tau}\left(\mathscr{U}_{1} \mathscr{U}_{2} f_{2}^{2}\right)(\theta)}{\left({ }^{\Psi} \mathscr{R}_{0}^{\kappa, \tau}\left\{\left(\mathscr{U}_{1} \mathscr{V}_{1}+\mathscr{U}_{2} \mathscr{V}_{2}\right) f_{1} f_{2}\right\}(\theta)\right)^{2}} \leq \frac{1}{4}
$$


Proof. From the given hypothesis, we have

$$
\left(\frac{\mathscr{U}_{2}(\vartheta)}{\mathscr{V}_{1}(\vartheta)}-\frac{f_{1}(\vartheta)}{f_{2}(\vartheta)}\right) \geq 0
$$

Similarly, we have

$$
\left(\frac{f_{1}(\vartheta)}{f_{2}(\vartheta)}-\frac{\mathcal{U}_{1}(\vartheta)}{\mathscr{V}_{2}(\vartheta)}\right) \geq 0
$$

Taking product of (22) and (23), we get

$$
\left(\frac{\mathcal{U}_{2}(\vartheta)}{\mathscr{V}_{1}(\vartheta)}-\frac{f_{1}(\vartheta)}{f_{2}(\vartheta)}\right)\left(\frac{f_{1}(\vartheta)}{f_{2}(\vartheta)}-\frac{\mathscr{U}_{1}(\vartheta)}{\mathscr{V}_{2}(\vartheta)}\right) \geq 0 .
$$

From (24), it can be written as

$$
\left(\mathscr{U}_{1}(\vartheta) \mathscr{V}_{1}(\vartheta)+\mathscr{U}_{2}(\vartheta) \mathscr{V}_{2}(\vartheta)\right) f_{1}(\vartheta) f_{2}(\vartheta) \geq \mathscr{V}_{1}(\vartheta) \mathscr{V}_{2}(\vartheta) f_{1}^{2}(\vartheta)+\mathscr{U}_{1}(\vartheta) \mathcal{U}_{2}(\vartheta) f_{2}^{2}(\vartheta)
$$

Now, taking product of (25) with $e^{-\tau(\Psi(\theta)-\Psi(\theta))}(\Psi(\theta)-\Psi(\theta))^{\kappa-1} \Psi^{\prime}(\theta) / \Gamma(\kappa)$ and integrating the resultant identity with respect to $\vartheta$ over $(0, \theta)$, we have

$$
\begin{aligned}
& \frac{1}{\Gamma(\kappa)} \int_{0}^{\theta} e^{-\tau(\Psi(\theta)-\Psi(\vartheta))}(\Psi(\theta)-\Psi(\vartheta))^{\kappa-1} \Psi^{\prime}(\vartheta)\left(\mathcal{U}_{1}(\vartheta) \mathscr{V}_{1}(\vartheta)+\mathscr{U}_{2}(\vartheta) \mathscr{V}_{2}(\vartheta)\right) f_{1}(\vartheta) f_{2}(\vartheta) \mathrm{d} \vartheta \\
& \quad \geq \frac{1}{\Gamma(\kappa)} \int_{0}^{\theta} e^{-\tau(\Psi(\theta)-\Psi(\vartheta))}(\Psi(\theta)-\Psi(\vartheta))^{\kappa-1} \Psi^{\prime}(\vartheta) \mathscr{V}_{1}(\vartheta) \mathscr{V}_{2}(\vartheta) f_{1}^{2}(\vartheta) \mathrm{d} \vartheta \\
& \quad+\frac{1}{\Gamma(\kappa)} \int_{0}^{\theta} e^{-\tau(\Psi(\theta)-\Psi(\vartheta))}(\Psi(\theta)-\Psi(\vartheta))^{\kappa-1} \Psi^{\prime}(\vartheta) \mathcal{U}_{1}(\vartheta) \mathcal{U}_{2}(\vartheta) f_{2}^{2}(\vartheta) \mathrm{d} \vartheta
\end{aligned}
$$

With the aid of Definition 8, we can write

$$
{ }^{\Psi} \mathscr{R}_{0}^{\kappa, \tau}\left[\left(\mathcal{U}_{1} \mathscr{V}_{1}+\mathscr{U}_{2} \mathscr{V}_{2}\right) f_{1} f_{2}\right](\theta) \geq{ }^{\Psi} \mathscr{R}_{0}^{\kappa, \tau}\left(\mathscr{V}_{1} \mathscr{V}_{2} f_{1}^{2}\right)(\theta)+{ }^{\Psi} \mathscr{R}_{0}^{\kappa, \tau}\left(\mathcal{U}_{1} \mathscr{U}_{2} f_{2}^{2}\right)(\theta)
$$

By applying AM-GM inequality, i.e., $x_{1}+x_{2} \geq$

$2 \sqrt{x_{1} x_{2}}, x_{1}, x_{2} \in \mathbb{R}^{+}$, we get

$$
{ }^{\Psi} \mathscr{R}_{0}^{\kappa, \tau}\left[\left(\mathcal{U}_{1} \mathscr{V}_{1}+\mathscr{U}_{2} \mathscr{V}_{2}\right) f_{1} f_{2}\right](\theta) \geq 2 \sqrt{\Psi \mathscr{R}_{0}^{\kappa, \tau}\left(\mathscr{V}_{1} \mathscr{V}_{2} f_{1}^{2}\right)(\theta)^{\Psi} \mathscr{R}_{0}^{\kappa, \tau}\left(\mathscr{U}_{1} \mathscr{U}_{2} f_{2}^{2}\right)(\theta)}
$$

It follows that

$$
\begin{aligned}
&{ }^{\Psi} \mathscr{R}_{0}^{\kappa, \tau}\left(\mathscr{V}_{1} \mathscr{V}_{2} f_{1}^{2}\right)(\theta)^{\Psi} \mathscr{R}_{0}^{\kappa, \tau}\left(\mathscr{U}_{1} \mathscr{U}_{2} f_{2}^{2}\right)(\theta) \\
& \quad \leq \frac{1}{4}\left({ }^{\Psi} \mathscr{R}_{0}^{\kappa, \tau}\left[\left(\mathcal{U}_{1} \mathscr{V}_{1}+\mathscr{U}_{2} \mathscr{V}_{2}\right) f_{1} f_{2}\right](\theta)\right)^{2},
\end{aligned}
$$

which gives the desired assertion (21).

Corollary 1. Let the functions $f_{1}$ and $f_{2}$ be positive and integrable on $[0, \infty)$ and assume that the function $\Psi$ is positive, monotone, and increasing on $[0, \infty[$, and its derivative $\Psi^{\prime}$ is continuous on $[0, \infty[$ with $\Psi(0)=0$. Suppose that $\mathscr{U}_{1}, \mathscr{U}_{2}, \mathscr{V}_{1}$, and $\mathscr{V}_{2}$ are four positive and integrable functions on $[0, \infty)$ such that

$$
\begin{aligned}
\left(H_{2}\right) 0 & <m_{1} \leq f_{1}(\vartheta) \leq M_{1}<\infty, 0<n_{1} \leq f_{2}(\vartheta) \leq N_{1}<\infty \\
\vartheta & \in[0, \theta], \theta>0 .
\end{aligned}
$$

Then, for $\kappa>0, \tau \geq 0$, and $\theta>0$, the following tempered fractional integral inequality holds:

$$
\begin{aligned}
& \frac{{ }^{\Psi} \mathscr{R}_{0}^{\kappa, \tau}\left(\mathscr{V}_{1} \mathscr{V}_{2} f_{1}^{2}\right)(\theta)^{\Psi} \mathscr{R}_{0}^{\kappa, \tau}\left(\mathscr{U}_{1} \mathscr{U}_{2} f_{2}^{2}\right)(\theta)}{\left({ }^{\Psi} \mathscr{R}_{0}^{\kappa, \tau}\left\{f_{1} f_{2}\right\}(\theta)\right)^{2}} \\
& \leq \frac{1}{4}\left(\sqrt{\frac{m_{1} n_{1}}{M_{1} N_{1}}}+\sqrt{\frac{M_{1} N_{1}}{m_{1} n_{1}}}\right)^{2} .
\end{aligned}
$$


Lemma 2. Let all the conditions of Lemma 1 hold. Then, for $\kappa, \lambda>0, \tau \geq 0$, and $\theta>0$, the following tempered fractional integral inequality holds:

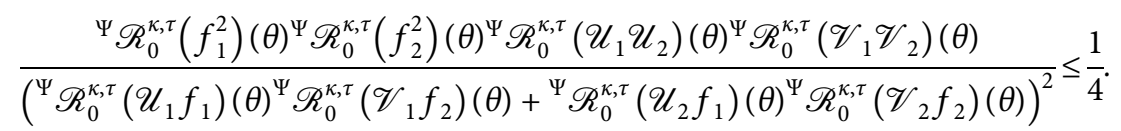

Proof. From the hypothesis $\left(H_{1}\right)$ defined by $(20)$, we have

$$
\begin{gathered}
\left(\frac{\mathscr{U}_{2}(\vartheta)}{\mathscr{V}_{1}(\zeta)}-\frac{f_{1}(\vartheta)}{f_{2}(\zeta)}\right) \geq 0, \\
\left(\frac{f_{1}(\vartheta)}{f_{2}(\zeta)}-\frac{\mathscr{U}_{1}(\vartheta)}{\mathscr{V}_{2}(\zeta)}\right) \geq 0,
\end{gathered}
$$

which follows that

$$
\left(\frac{\mathcal{U}_{1}(\vartheta)}{\mathscr{V}_{2}(\zeta)}+\frac{\mathcal{U}_{2}(\vartheta)}{\mathscr{V}_{1}(\zeta)}\right) \frac{f_{1}(\vartheta)}{f_{2}(\zeta)} \geq \frac{f_{1}^{2}(\vartheta)}{f_{2}^{2}(\zeta)}+\frac{\mathscr{U}_{1}(\vartheta) \mathcal{U}_{2}(\vartheta)}{\mathcal{U}_{1}(\zeta) \mathcal{U}_{2}(\zeta)}
$$

Taking product on both sides of (18) by $\mathscr{V}_{1}(\zeta) \mathscr{V}_{2}(\zeta) f_{2}^{2}(\zeta)$, we obtain

$$
\begin{aligned}
& \mathcal{U}_{1}(\vartheta) f_{1}(\vartheta) \mathscr{V}_{1}(\zeta) f_{2}(\zeta)+\mathscr{U}_{2}(\vartheta) f_{1}(\vartheta) \mathscr{V}_{2}(\zeta) f_{2}(\zeta) \\
& \geq \mathscr{V}_{1}(\zeta) \mathscr{V}_{2}(\zeta) f_{1}^{2}(\vartheta)+\mathcal{U}_{1}(\vartheta) \mathcal{U}_{2}(\vartheta) f_{2}^{2}(\zeta) .
\end{aligned}
$$

Taking product of both sides of (36) with $e^{-\tau(\Psi(\theta)-\Psi(\theta))}(\Psi(\theta)-\Psi(\theta))^{\kappa-1} \Psi^{\prime}(\theta) / \Gamma(\kappa)$ and integrating the resultant identity with respect to $\vartheta$ over $(0, \theta)$, we have

$$
\begin{aligned}
& \mathscr{V}_{1}(\zeta) f_{2}(\zeta) \frac{1}{\Gamma(\kappa)} \int_{0}^{\theta} e^{-\tau(\Psi(\theta)-\Psi(\vartheta))}(\Psi(\theta)-\Psi(\vartheta))^{\kappa-1} \Psi^{\prime}(\vartheta) \mathcal{U}_{1}(\vartheta) f_{1}(\vartheta) \mathrm{d} \vartheta \\
& \quad+\mathscr{V}_{2}(\zeta) f_{2}(\zeta) \frac{1}{\Gamma(\kappa)} \int_{0}^{\theta} e^{-\tau(\Psi(\theta)-\Psi(\vartheta))}(\Psi(\theta)-\Psi(\vartheta))^{\kappa-1} \Psi^{\prime}(\vartheta) \mathcal{U}_{2}(\vartheta) f_{1}(\vartheta) \mathrm{d} \vartheta \\
& \geq \mathscr{V}_{1}(\zeta) \mathscr{V}_{2}(\zeta) \frac{1}{\Gamma(\kappa)} \int_{0}^{\theta} e^{-\tau(\Psi(\theta)-\Psi(\vartheta))}(\Psi(\theta)-\Psi(\vartheta))^{\kappa-1} \Psi^{\prime}(\vartheta) f_{1}^{2}(\vartheta) \mathrm{d} \vartheta \\
& \quad+f_{2}^{2}(\zeta) \frac{1}{\Gamma(\kappa)} \int_{0}^{\theta} e^{-\tau(\Psi(\theta)-\Psi(\vartheta))}(\Psi(\theta)-\Psi(\vartheta))^{\kappa-1} \Psi^{\prime}(\vartheta) \mathcal{U}_{1}(\vartheta) \mathcal{U}_{2}(\vartheta) \mathrm{d} \vartheta
\end{aligned}
$$

which by applying (8) becomes

$$
\begin{array}{r}
\mathscr{V}_{1}(\zeta) f_{2}(\zeta)^{\Psi} \mathscr{R}_{0}^{\kappa, \tau}\left(\mathscr{U}_{1} f_{1}\right)(\theta)+\mathscr{V}_{2}(\zeta) f_{2}(\zeta)^{\Psi} \mathscr{R}_{0}^{\kappa, \tau}\left(\mathcal{U}_{2} f_{1}\right)(\theta) \\
\geq \mathscr{V}_{1}(\zeta) \mathscr{V}_{2}(\zeta)^{\Psi} \mathscr{R}_{0}^{\kappa, \tau}\left(f_{1}^{2}\right)(\theta)+f_{2}^{2}(\zeta)^{\Psi} \mathscr{R}_{0}^{\kappa, \tau}\left(\mathcal{U}_{1} \mathscr{U}_{2}\right)(\theta) .
\end{array}
$$

Again, taking product of both sides of (38) with $e^{-\tau(\Psi(\theta)-\Psi(\zeta))}(\Psi(\theta)-\Psi(\zeta))^{\lambda-1} \Psi^{\prime}(\zeta) / \Gamma(\lambda)$ and integrating the resultant identity with respect to $\zeta$ over $(0, \theta)$ and then applying (17), we get

$$
\begin{gathered}
{ }^{\Psi} \mathscr{R}_{0}^{\lambda, \tau}\left(\mathscr{V}_{1} f_{2}\right)(\theta)^{\Psi} \mathscr{R}_{0}^{\lambda, \tau}\left(\mathscr{U}_{1} f_{1}\right)(\theta)+{ }^{\Psi} \mathscr{R}_{0}^{\lambda, \tau}\left(\mathscr{V}_{2} f_{2}\right)(\theta)^{\Psi} \mathscr{R}_{0}^{\lambda, \tau}\left(\mathscr{U}_{2} f_{1}\right)(\theta) \\
\geq{ }^{\Psi} \mathscr{R}_{0}^{\lambda, \tau}\left(\mathscr{V}_{1} \mathscr{V}_{2}\right)(\theta)^{\Psi} \mathscr{R}_{0}^{\lambda, \tau}\left(f_{1}^{2}\right)(\theta)+{ }^{\Psi} \mathscr{R}_{0}^{\lambda, \tau}\left(f_{2}^{2}\right)(\theta)^{\Psi} \mathscr{R}_{0}^{\lambda, \tau}\left(\mathscr{U}_{1} \mathscr{U}_{2}\right)(\theta) .
\end{gathered}
$$

By using AM-GM inequality, we get

$$
\begin{gathered}
{ }^{\Psi} \mathscr{R}_{0}^{\lambda, \tau}\left(\mathscr{V}_{1} f_{2}\right)(\theta)^{\Psi} \mathscr{R}_{0}^{\lambda, \tau}\left(\mathscr{U}_{1} f_{1}\right)(\theta)+{ }^{\Psi} \mathscr{R}_{0}^{\lambda, \tau}\left(\mathscr{V}_{2} f_{2}\right)(\theta)^{\Psi} \mathscr{R}_{0}^{\lambda, \tau}\left(\mathscr{U}_{2} f_{1}\right)(\theta) \\
\geq 2 \sqrt{\Psi \mathscr{R}_{0}^{\lambda, \tau}\left(\mathscr{V}_{1} \mathscr{V}_{2}\right)(\theta)^{\Psi} \mathscr{R}_{0}^{\lambda, \tau}\left(f_{1}^{2}\right)(\theta)^{\Psi} \mathscr{R}_{0}^{\lambda, \tau}\left(f_{2}^{2}\right)(\theta)^{\Psi} \mathscr{R}_{0}^{\lambda, \tau}\left(\mathscr{U}_{1} \mathscr{U}_{2}\right)(\theta) .}
\end{gathered}
$$


It follows that

$$
\begin{aligned}
& { }^{\Psi} \mathscr{R}_{0}^{\lambda, \tau}\left(\mathscr{V}_{1} \mathscr{V}_{2}\right)(\theta)^{\Psi} \mathscr{R}_{0}^{\lambda, \tau}\left(f_{1}^{2}\right)(\theta)^{\Psi} \mathscr{R}_{0}^{\lambda, \tau}\left(f_{2}^{2}\right)(\theta)^{\Psi} \mathscr{R}_{0}^{\lambda, \tau}\left(\mathscr{U}_{1} \mathscr{U}_{2}\right)(\theta) \\
& \quad \leq \frac{1}{4}\left({ }^{\Psi} \mathscr{R}_{0}^{\lambda, \tau}\left(\mathscr{V}_{1} f_{2}\right)(\theta)^{\Psi} \mathscr{R}_{0}^{\lambda, \tau}\left(\mathscr{U}_{1} f_{1}\right)(\theta)+{ }^{\Psi} \mathscr{R}_{0}^{\lambda, \tau}\left(\mathscr{V}_{2} f_{2}\right)(\theta)^{\Psi} \mathscr{R}_{0}^{\lambda, \tau}\left(\mathcal{U}_{2} f_{1}\right)(\theta)\right)^{2},
\end{aligned}
$$

which completes the desired assertion (17).

$$
\begin{aligned}
& \frac{\gamma(\kappa, \tau \Psi(\theta)) \gamma(\kappa, \tau \Psi(\theta))}{\tau^{\kappa+\lambda} \Gamma(\kappa) \Gamma(\lambda)} \frac{{ }^{\Psi} \mathscr{R}_{0}^{\kappa, \tau}\left(f_{1}^{2}\right)(\theta)^{\Psi} \mathscr{R}_{0}^{\kappa, \tau}\left(f_{2}^{2}\right)(\theta)}{\left({ }^{\Psi} \mathscr{R}_{0}^{\kappa, \tau}\left(f_{1}\right)(\theta)^{\Psi} \mathscr{R}_{0}^{\kappa, \tau}\left(f_{2}\right)(\theta)\right)^{2}} \\
& \quad \leq \frac{1}{4}\left(\sqrt{\frac{m_{1} n_{1}}{M_{1} N_{1}}}+\sqrt{\frac{M_{1} N_{1}}{m_{1} n_{1}}}\right)^{2}
\end{aligned}
$$

on $[0, \infty[$ with $\Psi(0)=0$. Then, for $\kappa, \lambda>0, \tau \geq 0$, and $\theta>0$, the following tempered fractional integral inequality holds:

where

$$
\begin{aligned}
{ }^{\Psi} \mathscr{R}_{0}^{\kappa, \tau}[1] & =\frac{1}{\Gamma(\kappa)} \int_{0}^{\theta} \exp [-\tau(\Psi(\theta)-\Psi(\vartheta))](\Psi(\theta)-\Psi(\vartheta))^{\kappa-1} \Psi^{\prime}(\vartheta) \mathrm{d} \vartheta \\
& =\frac{1}{\Gamma(\kappa)} \int_{0}^{\Psi(\theta)} e^{-\tau z} z^{\kappa-1} \mathrm{~d} z,(\Psi(0)=0) \\
& =\frac{1}{\tau^{\kappa} \Gamma(\kappa)} \int_{0}^{\tau \Psi(\theta)} e^{-u} u^{\kappa-1} \mathrm{~d} u \\
& =\frac{\gamma(\kappa, \tau \Psi(\theta))}{\tau^{\kappa} \Gamma(\kappa)}
\end{aligned}
$$

and $\gamma(\kappa, t)=\int_{0}^{t} e^{-u} u^{\kappa-1} d u$ is the well-known incomplete gamma function (see [22]).

Lemma 3. Suppose that all the conditions of Lemma 1 hold and assume that the function $\Psi$ is positive, monotone, and increasing on $\left[0, \infty\left[\right.\right.$, and its derivative $\Psi^{\prime}$ is continuous on $[0, \infty[$ with $\Psi(0)=0$. Then, for $\kappa, \lambda>0, \tau \geq 0$, and $\theta>0$, the following tempered fractional integral inequality holds:

$$
{ }^{\Psi} \mathscr{R}_{0}^{\kappa, \tau}\left(f_{1}^{2}\right)(\theta)^{\Psi} \mathscr{R}_{0}^{\kappa, \tau}\left(f_{2}^{2}\right)(\theta) \leq{ }^{\Psi} \mathscr{R}_{0}^{\kappa, \tau}\left(\frac{\mathscr{U}_{2} f_{1} f_{2}}{\mathscr{V}_{1}}\right)(\theta){ }^{\Psi} \mathscr{R}_{0}^{\kappa, \tau}\left(\frac{\mathscr{V}_{2} f_{1} f_{2}}{\mathscr{U}_{1}}\right)(\theta) .
$$

Proof. From the hypothesis $\left(H_{1}\right)$ defined by $(20)$, we have

$$
\begin{aligned}
& \frac{1}{\Gamma(\kappa)} \int_{0}^{\theta} e^{-\tau(\Psi(\theta)-\Psi(\vartheta))}(\Psi(\theta)-\Psi(\vartheta))^{\kappa-1} \Psi^{\prime}(\vartheta) f_{1}^{2}(\vartheta) \mathrm{d} \vartheta \\
& \quad \leq \frac{1}{\Gamma(\kappa)} \int_{0}^{\theta} e^{-\tau(\Psi(\theta)-\Psi(\vartheta))}(\Psi(\theta)-\Psi(\vartheta))^{\kappa-1} \Psi^{\prime}(\vartheta) \frac{\mathscr{U}_{2}(\vartheta)}{\mathscr{V}_{1}(\vartheta)} f_{1}(\vartheta) f_{2}(\vartheta) \mathrm{d} \vartheta
\end{aligned}
$$


which in view of (17) yields

$$
{ }^{\Psi} \mathscr{R}_{0}^{\kappa, \tau}\left(f_{1}^{2}\right)(\theta) \leq{ }^{\Psi} \mathscr{R}_{0}^{\kappa, \tau}\left(\frac{\mathscr{U}_{2} f_{1} f_{2}}{\mathscr{V}_{1}}\right)(\theta) .
$$

Similarly, one can obtain

$$
{ }^{\Psi} \mathscr{R}_{0}^{\lambda, \tau}\left(f_{2}^{2}\right)(\theta) \leq{ }^{\Psi} \mathscr{R}_{0}^{\lambda, \tau}\left(\frac{\mathscr{V}_{2} f_{1} f_{2}}{\mathcal{U}_{1}}\right)(\theta) .
$$

Hence, the product of (46) and (47) yields the desired assertion (44).

Corollary 3. Let the functions $f_{1}$ and $f_{2}$ be positive and integrable on $[0, \infty)$ satisfying the hypothesis $\left(H_{2}\right)$ defined by (30) and assume that the function $\Psi$ is positive, monotone, and increasing on $\left[0, \infty\left[\right.\right.$, and its derivative $\Psi^{\prime}$ is continuous on $[0, \infty[$ with $\Psi(0)=0$. Then, for $\kappa, \lambda>0, \tau \geq 0$, and $\theta>0$, the following tempered fractional integral inequality holds:

$$
\frac{{ }^{\Psi} \mathscr{R}_{0}^{\kappa, \tau}\left(f_{1}^{2}\right)(\theta)^{\Psi} \mathscr{R}_{0}^{\kappa, \tau}\left(f_{2}^{2}\right)(\theta)}{\Psi \mathscr{R}_{0}^{\kappa, \tau}\left(f_{1} f_{2}\right)(\theta)^{\Psi} \mathscr{R}_{0}^{\kappa, \tau}\left(f_{1} f_{2}\right)(\theta)} \leq \frac{M_{1} N_{1}}{m_{1} n_{1}} .
$$

\section{Chebyshev-Type Tempered Fractional Integral Inequalities}

In this section, certain Chebyshev-type inequalities via tempered fractional integral (20) are presented with the help of Pólya-Szegö integral inequality given by Lemma 1 .

Theorem 1. Let the functions $f_{1}$ and $f_{2}$ be positive and integrable on $[0, \infty)$ and assume that the function $\Psi$ is

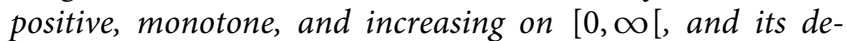
rivative $\Psi^{\prime}$ is continuous on $[0, \infty[$ with $\Psi(0)=0$. Suppose that $\mathscr{U}_{1}, \mathscr{U}_{2}, \mathscr{V}_{1}$, and $\mathscr{V}_{2}$ are four positive and integrable functions on $[0, \infty)$ satisfying the hypothesis $\left(H_{1}\right)$ defined by (10). Then, for $\kappa, \lambda>0, \tau \geq 0$, and $\theta>0$, the following tempered fractional integral inequality holds:

$$
\begin{aligned}
& \left|\frac{\gamma(\kappa, \tau \Psi(\theta))}{\tau^{\kappa} \Gamma(\kappa)}{ }^{\kappa} \mathscr{R}_{0}^{\kappa, \tau}\left(f_{1} f_{2}\right)(\theta)+\frac{\gamma(\lambda, \tau \Psi(\theta))^{\Psi}}{\tau^{\lambda} \Gamma(\lambda)} \mathscr{R}_{0}^{\lambda, \tau}\left(f_{1} f_{2}\right)(\theta)-{ }^{\Psi} \mathscr{R}_{0}^{\kappa, \tau}\left(f_{1}\right)(\theta)^{\Psi} \mathscr{R}_{0}^{\kappa, \tau}\left(f_{2}\right)(\theta)-{ }^{\Psi} \mathscr{R}_{0}^{\kappa, \tau}\left(f_{2}\right)(\theta)^{\Psi} \mathscr{R}_{0}^{\kappa, \tau}\left(f_{1}\right)(\theta)\right| \\
& \quad \leq\left|F_{1}\left(f_{1}, \mathscr{U}_{1}, \mathscr{U}_{2}\right)(\theta)+F_{2}\left(f_{1}, \mathscr{U}_{1}, \mathscr{U}_{2}\right)(\theta)\right|^{1 / 2} \times\left|F_{1}\left(f_{2}, \mathscr{V}_{1}, \mathscr{V}_{2}\right)(\theta)+F_{2}\left(f_{1}, \mathscr{V}_{1}, \mathscr{V}_{2}\right)(\theta)\right|^{1 / 2},
\end{aligned}
$$

where

$$
\begin{aligned}
& F_{1}\left(f_{1}, \mathscr{U}_{1}, \mathscr{U}_{2}\right)(\theta)=\frac{\gamma(\lambda, \tau \Psi(\theta))}{4 \tau^{\lambda} \Gamma(\lambda)} \frac{\left({ }^{\Psi} \mathscr{R}_{0}^{\kappa, \tau}\left\{\left(\mathcal{U}_{1}+\mathscr{U}_{2}\right) f_{1}\right\}\right)^{2}}{\Psi \mathscr{R}_{0}^{\kappa, \tau}\left(\mathcal{U}_{1} \mathcal{U}_{2}\right)(\theta)}-{ }^{\Psi} \mathscr{R}_{0}^{\kappa, \tau}\left(f_{1}\right)(\theta)^{\Psi} \mathscr{R}_{0}^{\kappa, \tau}\left(f_{1}\right)(\theta), \\
& F_{2}\left(f_{1}, \mathscr{U}_{1}, \mathscr{U}_{2}\right)(\theta)=\frac{\gamma(\kappa, \tau \Psi(\theta))}{4 \tau^{\kappa} \Gamma(\kappa)} \frac{\left({ }^{\Psi} \mathscr{R}_{0}^{\lambda, \tau}\left\{\left(\mathcal{U}_{1}+\mathscr{U}_{2}\right) f_{1}\right\}\right)^{2}}{\Psi \mathscr{R}_{0}^{\lambda, \tau}\left(\mathcal{U}_{1} \mathcal{U}_{2}\right)(\theta)}-{ }^{\Psi} \mathscr{R}_{0}^{\lambda, \tau}\left(f_{1}\right)(\theta)^{\Psi} \mathscr{R}_{0}^{\lambda, \tau}\left(f_{1}\right)(\theta) .
\end{aligned}
$$

Proof. By the given hypothesis, both the functions $f_{1}$ and $f_{2}$ are positive and integrable functions on $[0, \infty)$. Therefore, for $\vartheta, \zeta \in(0, \theta)$ with $\theta>0$, we define $\mathscr{A}(\vartheta, \zeta)$ by

$$
\begin{aligned}
\mathscr{A}(\vartheta, \zeta)= & \left(f_{1}(\vartheta)-f_{1}(\zeta)\right)\left(f_{2}(\vartheta)-f_{2}(\zeta)\right) \\
= & f_{1}(\vartheta) f_{2}(\vartheta)+f_{1}(\zeta) f_{2}(\zeta)-f_{1}(\vartheta) f_{2}(\zeta) \\
& -f_{1}(\zeta) f_{2}(\vartheta) .
\end{aligned}
$$

Multiplying (52) by $(1 / \Gamma(\kappa) \Gamma(\lambda)) \exp [-\tau(\Psi(\theta)-\Psi(\vartheta))]$ $\exp [-\tau(\Psi(\theta)-\Psi(\zeta))](\Psi(\theta)-\Psi(\vartheta))^{\kappa-1}(\Psi(\theta)-\Psi(\zeta))^{\lambda-1}$ $\Psi^{\prime}(\vartheta) \Psi^{\prime}(\zeta)$ and double integrating the resultant identity with respect to $\vartheta$ and $\zeta$ over $(0, \theta)$ and then using (17), we obtain

$$
\begin{aligned}
& \frac{1}{\Gamma(\kappa) \Gamma(\lambda)} \int_{0}^{\theta} \int_{0}^{\theta} \exp [-\tau(\Psi(\theta)-\Psi(\vartheta))] \exp [-\tau(\Psi(\theta)-\Psi(\zeta))] \\
& \quad \times(\Psi(\theta)-\Psi(\vartheta))^{\kappa-1}(\Psi(\theta)-\Psi(\zeta))^{\lambda-1} \Psi^{\prime}(\vartheta) \Psi^{\prime}(\zeta) \mathscr{A}(\vartheta, \zeta) \mathrm{d} \vartheta \mathrm{d} \zeta \\
& =\frac{\gamma(\kappa, \tau \Psi(\theta))}{\tau^{\kappa} \Gamma(\kappa)}{ }^{\Psi} \mathscr{R}_{0}^{\lambda, \tau}\left(f_{1} f_{2}\right)(\theta)+\frac{\gamma(\lambda, \tau \Psi(\theta))}{\tau^{\lambda} \Gamma(\lambda)}{ }^{\lambda} \mathscr{R}_{0}^{\lambda, \tau}\left(f_{1} f_{2}\right)(\theta) \\
& -{ }^{\Psi} \mathscr{R}_{0}^{\lambda, \tau}\left(f_{1}\right)(\theta)^{\Psi} \mathscr{R}_{0}^{\lambda, \tau}\left(f_{2}\right)(\theta)-{ }^{\Psi} \mathscr{R}_{0}^{\lambda, \tau}\left(f_{1}\right)(\theta)^{\Psi} \mathscr{R}_{0}^{\lambda, \tau}\left(f_{2}\right)(\theta) .
\end{aligned}
$$

By applying Cauchy-Schwartz inequality for double integrals, we have 


$$
\begin{aligned}
& \mid \frac{1}{\Gamma(\kappa) \Gamma(\lambda)} \int_{0}^{\theta} \int_{0}^{\theta} \exp [-\tau(\Psi(\theta)-\Psi(\vartheta))] \exp [-\tau(\Psi(\theta)-\Psi(\zeta))] \times(\Psi(\theta)-\Psi(\vartheta))^{\kappa-1} \\
& \cdot(\Psi(\theta)-\Psi(\zeta))^{\lambda-1} \Psi^{\prime}(\vartheta) \Psi^{\prime}(\zeta) \mathscr{A}(\vartheta, \zeta) \mathrm{d} \vartheta \mathrm{d} \zeta \mid \\
& \leq\left[\frac{1}{\Gamma(\kappa) \Gamma(\lambda)} \int_{0}^{\theta} \int_{0}^{\theta} \exp [-\tau(\Psi(\theta)-\Psi(\vartheta))] \exp [-\tau(\Psi(\theta)-\Psi(\zeta))]\right. \\
& \times(\Psi(\theta)-\Psi(\vartheta))^{\kappa-1}(\Psi(\theta)-\Psi(\zeta))^{\lambda-1} \Psi^{\prime}(\vartheta) \Psi^{\prime}(\zeta) f_{1}^{2}(\vartheta) \mathrm{d} \vartheta \mathrm{d} \zeta \\
& +\frac{1}{\Gamma(\kappa) \Gamma(\lambda)} \int_{0}^{\theta} \int_{0}^{\theta} \exp [-\tau(\Psi(\theta)-\Psi(\vartheta))] \exp [-\tau(\Psi(\theta)-\Psi(\zeta))] \\
& \times(\Psi(\theta)-\Psi(\vartheta))^{\kappa-1}(\Psi(\theta)-\Psi(\zeta))^{\lambda-1} \Psi^{\prime}(\vartheta) \Psi^{\prime}(\zeta) f_{1}^{2}(\zeta) \mathrm{d} \vartheta \mathrm{d} \zeta \\
& -2 \frac{1}{\Gamma(\kappa) \Gamma(\lambda)} \int_{0}^{\theta} \int_{0}^{\theta} \exp [-\tau(\Psi(\theta)-\Psi(\vartheta))] \exp [-\tau(\Psi(\theta)-\Psi(\zeta))] \\
& \left.\times(\Psi(\theta)-\Psi(\vartheta))^{\kappa-1}(\Psi(\theta)-\Psi(\zeta))^{\lambda-1} \Psi^{\prime}(\vartheta) \Psi^{\prime}(\zeta) f_{1}(\vartheta) f_{1}(\zeta) \mathrm{d} \vartheta \mathrm{d} \zeta\right]^{1 / 2} \\
& \times\left[\frac{1}{\Gamma(\kappa) \Gamma(\lambda)} \int_{0}^{\theta} \int_{0}^{\theta} \exp [-\tau(\Psi(\theta)-\Psi(\vartheta))] \exp [-\tau(\Psi(\theta)-\Psi(\zeta))]\right. \\
& \times(\Psi(\theta)-\Psi(\vartheta))^{\kappa-1}(\Psi(\theta)-\Psi(\zeta))^{\lambda-1} \Psi^{\prime}(\vartheta) \Psi^{\prime}(\zeta) f_{2}^{2}(\vartheta) \mathrm{d} \vartheta \mathrm{d} \zeta \\
& +\frac{1}{\Gamma(\kappa) \Gamma(\lambda)} \int_{0}^{\theta} \int_{0}^{\theta} \exp [-\tau(\Psi(\theta)-\Psi(\vartheta))] \exp [-\tau(\Psi(\theta)-\Psi(\zeta))] \\
& \times(\Psi(\theta)-\Psi(\vartheta))^{\kappa-1}(\Psi(\theta)-\Psi(\zeta))^{\lambda-1} \Psi^{\prime}(\vartheta) \Psi^{\prime}(\zeta) f_{2}^{2}(\zeta) \mathrm{d} \vartheta \mathrm{d} \zeta \\
& -2 \frac{1}{\Gamma(\kappa) \Gamma(\lambda)} \int_{0}^{\theta} \int_{0}^{\theta} \exp [-\tau(\Psi(\theta)-\Psi(\vartheta))] \exp [-\tau(\Psi(\theta)-\Psi(\zeta))] \\
& \left.\times(\Psi(\theta)-\Psi(\vartheta))^{\kappa-1}(\Psi(\theta)-\Psi(\zeta))^{\lambda-1} \Psi^{\prime}(\vartheta) \Psi^{\prime}(\zeta) f_{2}(\vartheta) f_{2}(\zeta) \mathrm{d} \vartheta \mathrm{d} \zeta\right]^{1 / 2}
\end{aligned}
$$

In view of (17) and (43), we get

$$
\begin{aligned}
& \mid \frac{1}{\Gamma(\kappa) \Gamma(\lambda)} \int_{0}^{\theta} \int_{0}^{\theta} \exp [-\tau(\Psi(\theta)-\Psi(\vartheta))] \exp [-\tau(\Psi(\theta)-\Psi(\zeta))] \times(\Psi(\theta)-\Psi(\vartheta))^{\kappa-1} \\
& \quad \cdot(\Psi(\theta)-\Psi(\zeta))^{\lambda-1} \Psi^{\prime}(\vartheta) \Psi^{\prime}(\vartheta) \mathscr{A}(\vartheta, \zeta) \mathrm{d} \vartheta \mathrm{d} \zeta \mid \\
& \leq\left[\frac{\gamma(\kappa, \tau \Psi(\theta))}{\tau^{\kappa} \Gamma(\kappa)} \mathscr{R}_{0}^{\lambda, \tau}\left(f_{1}^{2}\right)(\theta)+\frac{\gamma(\lambda, \tau \Psi(\theta))}{\tau^{\lambda} \Gamma(\lambda)} \mathscr{R}_{0}^{\lambda, \tau}\left(f_{1}^{2}\right)(\theta)-2^{\Psi} \mathscr{R}_{0}^{\lambda, \tau}\left(f_{1}\right)(\theta)^{\Psi} \mathscr{R}_{0}^{\lambda, \tau}\left(f_{1}\right)(\theta)\right]^{1 / 2} \\
& \quad \times\left[\frac{\gamma(\kappa, \tau \Psi(\theta))}{\tau^{\kappa} \Gamma(\kappa)}{ }^{\Psi / 2} \mathscr{R}_{0}^{\lambda, \tau}\left(f_{2}^{2}\right)(\theta)+\frac{\gamma(\lambda, \tau \Psi(\theta))}{\tau^{\lambda} \Gamma(\lambda)}{ }^{\prime} \mathscr{R}_{0}^{\lambda, \tau}\left(f_{2}^{2}\right)(\theta)-2^{\Psi} \mathscr{R}_{0}^{\lambda, \tau}\left(f_{2}\right)(\theta)^{\Psi} \mathscr{R}_{0}^{\lambda, \tau}\left(f_{2}\right)(\theta)\right]^{1 / 2} .
\end{aligned}
$$


Applying Lemma 1 for $\mathscr{V}_{1}(\theta)=\mathscr{V}_{2}(\theta)=f_{2}(\theta)=1$, we get

$$
\frac{\gamma(\lambda, \tau \Psi(\theta))}{\tau^{\lambda} \Gamma(\lambda)}{ }^{\Psi} \mathscr{R}_{0}^{\kappa, \tau}\left(f_{1}^{2}\right)(\theta) \leq \frac{\gamma(\lambda, \tau \Psi(\theta))}{4 \tau^{\lambda} \Gamma(\lambda)} \frac{\left({ }^{\Psi} \mathscr{R}_{0}^{\kappa, \tau}\left\{\left(\mathcal{U}_{1}+\mathscr{U}_{2}\right) f_{1}\right\}\right)^{2}}{{ }^{\Psi} \mathscr{R}_{0}^{\kappa, \tau}\left(\mathcal{U}_{1} \mathscr{U}_{2}\right)(\theta)} .
$$

It follows that

$$
\begin{aligned}
& \frac{\gamma(\lambda, \tau \Psi(\theta))}{\tau^{\lambda} \Gamma(\lambda)}{ }^{\Psi} \mathscr{R}_{0}^{\kappa, \tau}\left(f_{1}^{2}\right)(\theta)-{ }^{\Psi} \mathscr{R}_{0}^{\kappa, \tau}\left(f_{1}\right)(\theta)^{\Psi} \mathscr{R}_{0}^{\kappa, \tau}\left(f_{1}\right)(\theta) \\
& \leq \frac{\gamma(\lambda, \tau \Psi(\theta))}{4 \tau^{\lambda} \Gamma(\lambda)} \frac{\left.{ }^{\Psi} \mathscr{R}_{0}^{\kappa, \tau}\left\{\left(\mathscr{U}_{1}+\mathscr{U}_{2}\right) f_{1}\right\}\right)^{2}}{{ }^{\Psi} \mathscr{R}_{0}^{\kappa, \tau}\left(\mathcal{U}_{1} \mathscr{U}_{2}\right)(\theta)} \\
& \quad-{ }^{\Psi} \mathscr{R}_{0}^{\kappa, \tau}\left(f_{1}\right)(\theta)^{\Psi} \mathscr{R}_{0}^{\kappa, \tau}\left(f_{1}\right)(\theta) \\
& =F_{1}\left(f_{1}, \mathscr{U}_{1}, \mathscr{U}_{2}\right)(\theta) .
\end{aligned}
$$

Similarly, one can get

$$
\begin{aligned}
& \frac{\gamma(\kappa, \tau \Psi(\theta))}{\tau^{\kappa} \Gamma(\kappa)}{ }^{\Psi} \mathscr{R}_{0}^{\lambda, \tau}\left(f_{1}^{2}\right)(\theta)-{ }^{\Psi} \mathscr{R}_{0}^{\lambda, \tau}\left(f_{1}\right)(\theta)^{\Psi} \mathscr{R}_{0}^{\lambda, \tau}\left(f_{1}\right)(\theta) \\
& \leq \frac{\gamma(\kappa, \tau \Psi(\theta))}{4 \tau^{\kappa} \Gamma(\kappa)} \frac{\left({ }^{\Psi} \mathscr{R}_{0}^{\lambda, \tau}\left\{\left(\mathscr{U}_{1}+\mathscr{U}_{2}\right) f_{1}\right\}\right)^{2}}{\Psi \mathscr{R}_{0}^{\lambda, \tau}\left(\mathcal{U}_{1} \mathscr{U}_{2}\right)(\theta)} \\
& \quad-{ }^{\Psi} \mathscr{R}_{0}^{\lambda, \tau}\left(f_{1}\right)(\theta)^{\Psi} \mathscr{R}_{0}^{\lambda, \tau}\left(f_{1}\right)(\theta) \\
& =F_{2}\left(f_{1}, \mathscr{U}_{1}, \mathscr{U}_{2}\right)(\theta) .
\end{aligned}
$$

Again applying Lemma 1 for $\mathscr{U}_{1}(\theta)=\mathscr{U}_{2}(\theta)=$ $f_{1}(\theta)=1$, we get

$$
\begin{aligned}
& \frac{\gamma(\lambda, \tau \Psi(\theta))}{\tau^{\lambda} \Gamma(\lambda)}{ }^{\Psi} \mathscr{R}_{0}^{\kappa, \tau}\left(f_{2}^{2}\right)(\theta)-{ }^{\Psi} \mathscr{R}_{0}^{\kappa, \tau}\left(f_{2}\right)(\theta)^{\Psi} \mathscr{R}_{0}^{\kappa, \tau}\left(f_{2}\right)(\theta) \\
& \leq \frac{\gamma(\lambda, \tau \Psi(\theta))}{4 \tau^{\lambda} \Gamma(\lambda)} \frac{\left.{ }^{\Psi} \mathscr{R}_{0}^{\kappa, \tau}\left\{\left(\mathscr{V}_{1}+\mathscr{V}_{2}\right) f_{2}\right\}\right)^{2}}{\Psi \mathscr{R}_{0}^{\kappa, \tau}\left(\mathscr{V}_{1} \mathscr{V}_{2}\right)(\theta)} \\
& \quad-{ }^{\Psi} \mathscr{R}_{0}^{\kappa, \tau}\left(f_{2}\right)(\theta)^{\Psi} \mathscr{R}_{0}^{\kappa, \tau}\left(f_{2}\right)(\theta) \\
& =F_{1}\left(f_{2}, \mathscr{U}_{1}, \mathscr{U}_{2}\right)(\theta),
\end{aligned}
$$

$$
\begin{aligned}
\frac{\gamma(\kappa, \tau \Psi(\theta))}{\tau^{\kappa} \Gamma(\kappa)}{ }^{\Psi} \mathscr{R}_{0}^{\lambda, \tau}\left(f_{2}^{2}\right)(\theta)-{ }^{\Psi} \mathscr{R}_{0}^{\lambda, \tau}\left(f_{2}\right)(\theta)^{\Psi} \mathscr{R}_{0}^{\lambda, \tau}\left(f_{2}\right)(\theta) \\
\leq \frac{\gamma(\kappa, \tau \Psi(\theta))}{4 \tau^{\kappa} \Gamma(\kappa)} \frac{\left({ }^{\Psi} \mathscr{R}_{0}^{\lambda, \tau}\left\{\left(\mathscr{V}_{1}+\mathscr{V}_{2}\right) f_{2}\right\}\right)^{2}}{\Psi \mathscr{R}_{0}^{\lambda, \tau}\left(\mathscr{V}_{1} \mathscr{V}_{2}\right)(\theta)} \\
\quad-{ }^{\Psi} \mathscr{R}_{0}^{\lambda, \tau}\left(f_{2}\right)(\theta){ }^{\Psi} \mathscr{R}_{0}^{\lambda, \tau}\left(f_{2}\right)(\theta) \\
=F_{2}\left(f_{2}, \mathscr{U}_{1}, \mathscr{U}_{2}\right)(\theta) .
\end{aligned}
$$

Thus, by considering (53) to (60), we arrive at the desired assertion (49). This is the desired proof of Theorem 1.

Theorem 2. Suppose that all the conditions of Theorem 1 are satisfied. Then, for $\kappa>0, \tau \geq 0$, and $\theta>0$, the following tempered fractional integral inequality holds:

$$
\begin{aligned}
& \left|\frac{\gamma(\kappa, \tau \Psi(\theta))}{\tau^{\kappa} \Gamma(\kappa)}{ }^{\Psi} \mathscr{R}_{0}^{\kappa, \tau}\left(f_{1} f_{2}\right)(\theta)-{ }^{\Psi} \mathscr{R}_{0}^{\kappa, \tau}\left(f_{1}\right)(\theta){ }^{\Psi} \mathscr{R}_{0}^{\kappa, \tau}\left(f_{2}\right)(\theta)\right| \\
& \quad \leq\left|F\left(f_{1}, \mathscr{U}_{1}, \mathscr{U}_{2}\right)(\theta) F\left(f_{2}, \mathscr{V}_{1}, \mathscr{V}_{2}\right)(\theta)\right|^{1 / 2},
\end{aligned}
$$

where

$$
\begin{aligned}
F\left(f_{1}, \mathscr{U}_{1}, \mathscr{U}_{2}\right)(\theta)= & \frac{\gamma(\kappa, \tau \Psi(\theta))}{4 \tau^{\kappa} \Gamma(\kappa)} \frac{\left({ }^{\Psi} \mathscr{R}_{0}^{\kappa, \tau}\left\{\left(\mathcal{U}_{1}+\mathscr{U}_{2}\right) f_{1}\right\}\right)^{2}}{{ }^{\Psi} \mathscr{R}_{0}^{\kappa, \tau}\left(\mathcal{U}_{1} \mathcal{U}_{2}\right)(\theta)} \\
& -\left({ }^{\Psi} \mathscr{R}_{0}^{\kappa, \tau}\left(f_{1}\right)(\theta)\right)^{2} .
\end{aligned}
$$

Proof. Applying Theorem 1 for $\kappa=\lambda$, we get the desired result in (61).

Remark 4. If we consider $\mathscr{U}_{1}=m_{1}, \mathscr{U}_{2}=M_{1}, \mathscr{V}_{1}=n_{1}$, and $\mathscr{V}_{2}=N_{1}$, then we have

$$
\begin{aligned}
& F\left(f_{1}, m_{1}, M_{1}\right)(\theta)=\frac{\left(M_{1}-m_{1}\right)^{2}}{4 M_{1} m_{1}}\left({ }^{\Psi} \mathscr{R}_{0}^{\kappa, \tau}\left(f_{1}\right)(\theta)\right)^{2}, \\
& F\left(f_{2}, m_{1}, M_{1}\right)(\theta)=\frac{\left(N_{1}-n_{1}\right)^{2}}{4 N_{1} n_{1}}\left({ }^{\Psi} \mathscr{R}_{0}^{\kappa, \tau}\left(f_{2}\right)(\theta)\right)^{2} .
\end{aligned}
$$

Corollary 4. Let the functions $f_{1}$ and $f_{2}$ be positive and integrable on $[0, \infty)$ and satisfying the hypothesis $\left(\mathrm{H}_{2}\right)$ given by (30). Then, for $\kappa>0, \tau \geq 0$, and $\theta>0$, the following tempered fractional integral inequality holds:

$$
\begin{aligned}
& \left|\frac{\gamma(\kappa, \tau \Psi(\theta))}{\tau^{\kappa} \Gamma(\kappa)}{ }^{\Psi} \mathscr{R}_{0}^{\kappa, \tau}\left(f_{1} f_{2}\right)(\theta)-{ }^{\Psi} \mathscr{R}_{0}^{\kappa, \tau}\left(f_{1}\right)(\theta)^{\Psi} \mathscr{R}_{0}^{\kappa, \tau}\left(f_{2}\right)(\theta)\right| \\
& \leq \frac{\left(M_{1}-m_{1}\right)\left(N_{1}-n_{1}\right)}{4 \sqrt{m_{1} M_{1} n_{1} N_{1}}}{ }^{\Psi} \mathscr{R}_{0}^{\kappa, \tau}\left(f_{1}\right)(\theta)^{\Psi} \mathscr{R}_{0}^{\kappa, \tau}\left(f_{2}\right)(\theta) .
\end{aligned}
$$




\section{Particular Cases}

The following new Pólya-Szegö- and Chebyshev-type inequalities for classical tempered fractional integral (14) can be easily established.

Lemma 4. Let the functions $f_{1}$ and $f_{2}$ be positive and integrable on $[0, \infty)$. Suppose that $\mathscr{U}_{1}, \mathscr{U}_{2}, \mathscr{V}_{1}$, and $\mathscr{V}_{2}$ are four positive and integrable functions on $[0, \infty)$ satisfying the hypothesis $\left(H_{1}\right)$ defined by (20). Then, for $\kappa>0, \tau \geq 0$, and $\theta>0$, the following tempered fractional integral inequality holds:

$$
\frac{\mathscr{R}_{0}^{\kappa, \tau}\left(\mathscr{V}_{1} \mathscr{V}_{2} f_{1}^{2}\right)(\theta) \mathscr{R}_{0}^{\kappa, \tau}\left(\mathscr{U}_{1} \mathscr{U}_{2} f_{2}^{2}\right)(\theta)}{\left(\mathscr{R}_{0}^{\kappa, \tau}\left\{\left(\mathscr{U}_{1} \mathscr{V}_{1}+\mathscr{U}_{2} \mathscr{V}_{2}\right) f_{1} f_{2}\right\}(\theta)\right)^{2}} \leq \frac{1}{4}
$$

Proof. Applying Lemma 1 for $\Psi(\theta)=\theta$, we get Lemma 4 .

Lemma 5. Let all the conditions of Lemma 4 are satisfied. Then, for $\kappa, \lambda>0, \tau \geq 0$, and $\theta>0$, the following tempered fractional integral inequality holds:

$$
\frac{\mathscr{R}_{0}^{\kappa, \tau}\left(f_{1}^{2}\right)(\theta) \mathscr{R}_{0}^{\lambda, \tau}\left(f_{2}^{2}\right)(\theta) \mathscr{R}_{0}^{\kappa, \tau}\left(\mathscr{U}_{1} \mathscr{U}_{2}\right)(\theta) \mathscr{R}_{0}^{\lambda, \tau}\left(\mathscr{V}_{1} \mathscr{V}_{2}\right)(\theta)}{\left(\mathscr{R}_{0}^{\kappa, \tau}\left(\mathscr{U}_{1} f_{1}\right)(\theta) \mathscr{R}_{0}^{\lambda, \tau}\left(\mathscr{V}_{1} f_{2}\right)(\theta)+\mathscr{R}_{0}^{\kappa, \tau}\left(\mathscr{U}_{2} f_{1}\right)(\theta) \mathscr{R}_{0}^{\lambda, \tau}\left(\mathscr{V}_{2} f_{2}\right)(\theta)\right)^{2}} \leq \frac{1}{4} .
$$

Proof. Applying Lemma 2 for $\Psi(\theta)=\theta$, we get Lemma 5.

Similarly, we can derive the particular case of Lemma 3. The following theorem represents the particular case of Theorem 1 in terms of classical tempered fractional integral.
Theorem 3. Let the functions $f_{1}$ and $f_{2}$ be positive and integrable on $[0, \infty)$. Suppose that $\mathscr{U}_{1}, \mathscr{U}_{2}, \mathscr{V}_{1}$, and $\mathscr{V}_{2}$ are four positive and integrable functions on $[0, \infty)$ satisfying the hypothesis $\left(H_{1}\right)$ defined by (20). Then, for $\kappa, \lambda>0, \tau \geq 0$, and $\theta>0$, the following tempered fractional integral inequality holds:

$$
\begin{aligned}
& \left|\frac{\gamma(\kappa, \tau \theta)}{\tau^{\kappa} \Gamma(\kappa)} \mathscr{R}_{0}^{\kappa, \tau}\left(f_{1} f_{2}\right)(\theta)+\frac{\gamma(\lambda, \tau \theta)}{\tau^{\lambda} \Gamma(\lambda)} \mathscr{R}_{0}^{\lambda, \tau}\left(f_{1} f_{2}\right)(\theta)-\mathscr{R}_{0}^{\kappa, \tau}\left(f_{1}\right)(\theta) \mathscr{R}_{0}^{\lambda, \tau}\left(f_{2}\right)(\theta)-\mathscr{R}_{0}^{\kappa, \tau}\left(f_{2}\right)(\theta) \mathscr{R}_{0}^{\lambda, \tau}\left(f_{1}\right)(\theta)\right| \\
& \quad \leq\left|F_{1}\left(f_{1}, \mathscr{U}_{1}, \mathscr{U}_{2}\right)(\theta)+F_{2}\left(f_{1}, \mathscr{U}_{1}, \mathscr{U}_{2}\right)(\theta)\right|^{1 / 2} \times\left|F_{1}\left(f_{2}, \mathscr{V}_{1}, \mathscr{V}_{2}\right)(\theta)+F_{2}\left(f_{1}, \mathscr{V}_{1}, \mathscr{V}_{2}\right)(\theta)\right|^{1 / 2},
\end{aligned}
$$

where

$$
\begin{aligned}
& F_{1}\left(f_{1}, \mathscr{U}_{1}, \mathscr{U}_{2}\right)(\theta)=\frac{\gamma(\lambda, \tau \theta)}{4 \tau^{\lambda} \Gamma(\lambda)} \frac{\left(\mathscr{R}_{0}^{\kappa, \tau}\left\{\left(\mathscr{U}_{1}+\mathscr{U}_{2}\right) f_{1}\right\}\right)^{2}}{\mathscr{R}_{0}^{\kappa, \tau}\left(\mathscr{U}_{1} \mathscr{U}_{2}\right)(\theta)}-\mathscr{R}_{0}^{\kappa, \tau}\left(f_{1}\right)(\theta) \mathscr{R}_{0}^{\lambda, \tau}\left(f_{1}\right)(\theta), \\
& F_{2}\left(f_{1}, \mathscr{U}_{1}, \mathscr{U}_{2}\right)(\theta)=\frac{\gamma(\kappa, \tau \theta)}{4 \tau^{\kappa} \Gamma(\kappa)} \frac{\left(\mathscr{R}_{0}^{\lambda, \tau}\left\{\left(\mathscr{U}_{1}+\mathscr{U}_{2}\right) f_{1}\right\}\right)^{2}}{\mathscr{R}_{0}^{\lambda, \tau}\left(\mathscr{U}_{1} \mathscr{U}_{2}\right)(\theta)}-\mathscr{R}_{0}^{\kappa, \tau}\left(f_{1}\right)(\theta) \mathscr{R}_{0}^{\lambda, \tau}\left(f_{1}\right)(\theta) .
\end{aligned}
$$

Proof. Applying Theorem 1 for $\Psi(\theta)=\theta$, then we get the desired Theorem 3.

Similarly, we can derive particular result of Theorem 2.

\section{Applications}

In this section, we define a way for constructing four bounded functions and then utilize them to present certain estimates of Chebyshev-type tempered fractional integral inequalities of two unknown functions.
Let the unit function $\hbar(\theta)$ be defined by

$$
\hbar(\theta)=\left\{\begin{array}{l}
1, \theta>0 \\
0, \theta \leq 0
\end{array}\right.
$$

and let the Heaviside unit step function $\hbar_{a}(\theta)$ be defined by

$$
\hbar_{a}(\theta)=\left\{\begin{array}{l}
1, \theta>a, \\
0, \theta \leq a .
\end{array}\right.
$$

Suppose that the function $\mathscr{U}_{1}$ is piecewise continuous function on $[0, X]$ defined by 


$$
\begin{aligned}
& \mathscr{U}_{1}(x)=m_{1_{1}}\left(\hbar_{0}(x)-\hbar_{x_{1}}(x)\right)+m_{1_{2}}\left(\hbar_{x_{1}}(x)-\hbar_{x_{2}}(x)\right)+\cdots+m_{1_{p+1}} \hbar_{x_{p}}(x) \\
& =m_{1_{1}} \hbar_{0}(x)+\left(m_{1_{2}}-m_{1_{1}}\right) \hbar_{x_{1}}(x)+\left(m_{1_{3}}-m_{1_{2}}\right) \hbar_{x_{2}}(x)+\cdots+\left(m_{1_{p+1}}-m_{1_{p}}\right) \hbar_{x_{p}}(x) \\
& =\sum_{i=0}^{p}\left(m_{1_{i+1}}-m_{1_{i}}\right) \hbar_{x_{i}}(x),
\end{aligned}
$$

where $m_{1_{0}}=0$ and $0=x_{0}<x_{1}<x_{2}<\cdots<x_{p}<x_{p+1}=X$. Similarly, we define

$$
\begin{aligned}
& \mathcal{U}_{2}(x)=\sum_{i=0}^{p}\left(M_{1_{i+1}}-M_{1_{i}}\right) \hbar_{x_{i}}(x), \\
& \mathscr{V}_{1}(x)=\sum_{i=0}^{p}\left(n_{1_{i+1}}-n_{1_{i}}\right) \hbar_{x_{i}}(x), \\
& \mathscr{V}_{2}(x)=\sum_{i=0}^{p}\left(N_{1_{i+1}}-N_{1_{i}}\right) \hbar_{x_{i}}(x),
\end{aligned}
$$

where the constants $n_{1_{0}}=N_{1_{0}}=M_{1_{0}}=0$. If there exists an integrable function $f_{1}$ on $[0, X]$ satisfying the hypothesis $\left(H_{1}\right)$, then we have $m_{1_{i+1}} \leq f_{1}(x) \leq M_{1_{i+1}}$ for each $x \in\left(x_{i}, x_{i+1}\right], i=0,1,2, \ldots, p$.

Proposition 1. Let the functions $f_{1}$ and $f_{2}$ be two positive and integrable on $[0, X]$. Assume that the functions $\mathscr{U}_{1}, \mathscr{U}_{2}$, $\mathscr{V}_{1}$, and $\mathscr{V}_{2}$ are defined by (73)-(76), respectively, and satisfying the hypothesis $\left(H_{1}\right)$ defined by (30). Then, for $\kappa>0$, the following inequality for tempered fractional integral holds:

$$
\begin{aligned}
& \left(\sum_{i=0}^{p} n_{1_{i+1}} N_{1_{i+1}} \Psi \mathscr{R}_{x_{i}, x_{i+1}}^{\kappa, \tau}\left(f_{1}^{2}\right)(X)\right)^{2}\left(\sum_{i=0}^{p} m_{1_{i+1}} M_{1_{i+1}} \Psi \mathscr{R}_{x_{i}, x_{i+1}}^{\kappa, \tau}\left(f_{2}^{2}\right)(X)\right)^{2} \\
& \quad \leq \frac{1}{4} \sum_{i=0}^{p}\left(n_{1_{i+1}} N_{1_{i+1}}+m_{1_{i+1}} M_{1_{i+1}}\right)^{\Psi} \mathscr{R}_{x_{i}, x_{i+1}}^{\kappa, \tau}\left(f_{1} f_{2}\right)(X) .
\end{aligned}
$$

Proof. By applying the Definition 8 , we have

$$
\begin{gathered}
{ }^{\Psi} \mathscr{R}_{0, X}^{\kappa, \tau}\left(\mathscr{V}_{1} \mathscr{V}_{2} f_{1}^{2}\right)(X)=\sum_{i=0}^{p} n_{1_{i+1}} N_{1_{i+1}}{ }^{\Psi} \mathscr{R}_{x_{i}, x_{i+1}}^{\kappa, \tau}\left(f_{1}^{2}\right)(X), \\
{ }^{\Psi} \mathscr{R}_{0, X}^{\kappa, \tau}\left(\mathscr{U}_{1} \mathscr{U}_{2} f_{2}^{2}\right)(X)=\sum_{i=0}^{p} m_{1_{i+1}} M_{1_{i+1}}{ }^{\Psi} \mathscr{R}_{x_{i}, x_{i+1}}^{\kappa, \tau}\left(f_{2}^{2}\right)(X), \\
{ }^{\Psi} \mathscr{R}_{0, X}^{\kappa, \tau}\left\{\left(\mathscr{U}_{1} \mathscr{V}_{1}+\mathscr{U}_{2} \mathscr{V}_{2}\right) f_{1} f_{2}\right\}(X)=\sum_{i=0}^{p}\left(m_{1_{i+1}} n_{1_{i+1}}+M_{1_{i+1}} N_{1_{i+1}}\right)^{\Psi} \mathscr{R}_{x_{i}, x_{i+1}}^{\kappa, \tau}\left(f_{1} f_{2}\right)(X) .
\end{gathered}
$$

Hence, by applying Lemma 1, we get the desired assertion (77).
Proposition 2. By setting $\Psi(\theta)=\theta$ in Proposition 1, then we arrive to the following result in terms of classical tempered fractional integral:

$$
\begin{aligned}
& \left(\sum_{i=0}^{p} n_{1_{i+1}} N_{1_{i+1}} \mathscr{R}_{x_{i}, x_{i+1}}^{\kappa, \tau}\left(f_{1}^{2}\right)(X)\right)^{2}\left(\sum_{i=0}^{p} m_{1_{i+1}} M_{1_{i+1}} \mathscr{R}_{x_{i}, x_{i+1}}^{\kappa, \tau}\left(f_{2}^{2}\right)(X)\right)^{2} \\
& \quad \leq \frac{1}{4} \sum_{i=0}^{p}\left(n_{1_{i+1}} N_{1_{i+1}}+m_{1_{i+1}} M_{1_{i+1}}\right) \mathscr{R}_{x_{i}, x_{i+1}}^{\kappa, \tau}\left(f_{1} f_{2}\right)(X) .
\end{aligned}
$$


Remark 5. Throughout in the paper, if we apply $\Psi(\theta)=\theta$ and $\tau=0$, then all the newly presented inequalities will be reduced to the work derived earlier by Ntouyas et al. [18].

\section{Concluding Remarks}

Certain new Pólya-Szegö- and Chebyshev-type inequalities by utilizing tempered fractional integral are presented in this paper. These inequalities generalized the existing inequalities. We can easily get the said Pólya-Szegö- and Chebyshev-type inequalities for Katugampola, generalized Riemann-Liouville, classical Riemann-Liouville, generalized conformable, and conformable fractional integrals by applying different conditions on function $\Psi$ given in Remark 2.

\section{Data Availability}

No data were used to support this study.

\section{Conflicts of Interest}

The authors declare that they have no conflicts of interest.

\section{Authors' Contributions}

All the authors contributed equally, and they have read and approved the final manuscript for publication.

\section{Acknowledgments}

Thabet Abdeljawad would like to thank Prince Sultan University for the support through the research group: Nonlinear Analysis Methods in Applied Mathematics (NAMAM) (group number RG-DES-2017-01-17).

\section{References}

[1] P. L. Chebyshev, "Sur les expressions approximatives des integrales definies par les autres prises entre les mêmes limites," Kharkov Mathematical Society, vol. 2, pp. 93-98, 1882.

[2] S. Belarbi and Z. Dahmani, "On some new fractional integral inequalities," Journal of Inequalities in Pure and Applied Mathematics, vol. 10, no. 3, pp. 1-12, 2009.

[3] S. S. Dragomir, "A generalization of Gruss's inequality in inner product spaces and applications," Journal of Mathematical Analysis and Applications, vol. 237, no. 1, pp. 74-82, 1999.

[4] Z. Dahmani, "About some integral inequalities using Riemann-Liouville integrals," General Mathematics, vol. 20, no. 4, pp. 63-69, 2012.

[5] S. L. Kalla and A. Rao, "On Grüss type inequality for hypergeometric fractional integrals," Matematiche (Catania), vol. 66, no. 1, pp. 57-64, 2011.

[6] A. Tassaddiq, G. Rahman, K. S. Nisar, and M. Samraiz, "Certain fractional conformable inequalities for the weighted and the extended Chebyshev functionals," Advances in Difference Equations, vol. 96, p. 2020, 2020.

[7] G. Grüss, "Uber, das Maximum des absoluten Betrages von $1 / b-a \int_{a}^{b}$
$\mathcal{U}(\varrho) \mathscr{V}(\varrho) \mathrm{d} \varrho-1 /(b-a)^{2} \int_{a}^{b} \mathcal{U}(\varrho) \mathrm{d} \varrho \int_{a}^{b} \mathscr{V}(\varrho) \mathrm{d} \varrho, "$ Mathematische Zeitschrift, vol. 39, pp. 215-226, 1935.

[8] G. Pólya and G. Szegö, Aufgaben und Lehrsatze aus der analysis, Band 1, Die Grundlehren der mathematischen Wissenschaften 19, Springer, Berlin, Germany, 1925.

[9] S. S. Dragomir and N. T. Diamond, "Integral inequalities of Grüss type via pólya-szegö and shisha-Mond results, east," Asian Journal of Mathematics, vol. 19, no. 1, pp. 27-39, 2003.

[10] Z. Dahmani and L. Tabharit, "On weighted gruss type inequalities via fractional integrals," Journal of Advanced Research in Pure Mathematics, vol. 2, no. 4, pp. 31-38, 2010.

[11] Z. Dahmani, "New inequalities in fractional integrals," International Journal of Nonlinear Science, vol. 9, pp. 493-497, 2010.

[12] M. Z. Sarikaya, Z. Dahmani, M. E. Kiris, and F. Ahmad, " $(k, s)$-Riemann-Liouville fractional integral and applications," Hacettepe Journal of Mathematics and Statistics, vol. 45, no. 1, pp. 77-89, 2016.

[13] E. Set, M. Tomar, and M. Z. Sarikaya, "On generalized Grüss type inequalities for k-fractional integrals," Applied Mathematics and Computation, vol. 269, pp. 29-34, 2015.

[14] F. Jarad, E. Ugurlu, T. Abdeljawad, and D. Baleanu, "On a new class of fractional operators," Advances in Difference Equations, vol. 2017, p. 247, 2017.

[15] F. Jarad, T. Abdeljawad, and J. Alzabut, "Generalized fractional derivatives generated by a class of local proportional derivatives," The European Physical Journal Special Topics, vol. 226, no. 16-18, pp. 3457-3471, 2017.

[16] C. J. Huang, G. Rahman, K. S. Nisar, A. Ghaffar, and F. Qi, "Some Inequalities of Hermite-Hadamard type for $k$-fractional conformable integrals," Australian Journal of Mathematical Analysis and Applications, vol. 16, no. 1, pp. 1-9, 2019.

[17] F. Qi, G. Rahman, S. Hussain, W.-S. Du, and K. Nisar, "Some inequalities of Čebyšev type for conformable $k$-fractional integral operators," Symmetry, vol. 10, no. 11, p. 614, 2018.

[18] S. K. Ntouyas, P. Agarwal, and J. Tariboon, "On Pólya-Szegö and Chebyshev types inequalities involving integral operators," Journal of Mathematical Inequalities, vol. 10, pp. 491-504, 2020.

[19] R. G. Buschman, "Decomposition of an integral operator by use of Mikusenski calculus," SIAM Journal of Mathematical Analysis, vol. 3, no. 1, pp. 83-85, 1972.

[20] C. Li, W. Deng, W. Deng, and L. Zhao, "Well-posedness and numerical algorithm for the tempered fractional differential equations," Discrete \& Continuous Dynamical Systems-B, vol. 24, no. 4, pp. 1989-2015, 2019.

[21] M. M. Meerschaert, F. Sabzikar, and J. Chen, "Tempered fractional calculus," Journal of Computational Physics, vol. 293, pp. 14-28, 2015.

[22] A. Fernandez and C. Ustaoğlu, "On some analytic properties of tempered fractional calculus," Journal of Computational and Applied Mathematics, vol. 366, 2020.

[23] H. M. Fahad, A. Fernandez, M. U. Rehman, and M. Siddiqi, "Tempered and hadamard-type fractional calculus with respect to functions," 2019.

[24] U. N. Katugampola, "New approach to a generalized fractional integral," Applied Mathematics and Computation, vol. 218, no. 3, pp. 860-865, 2011.

[25] H. Yildirim and Z. Kirtay, "Ostrowski inequality for generalized fractional integral and related inequalities," Malaya Journal of Matematik, vol. 2, pp. 322-329, 2014.

[26] E. Kacar, Z. Kacar, and H. Yildirim, "Integral inequalities for Riemann-Liouville fractional integrals of a function with respect to another function, Iran," Journal of Informatics and Mathematical Sciences, vol. 13, pp. 1-13, 2018. 
[27] A. A. Kilbas, H. M. Sarivastava, and J. J. Trujillo, Theory and Application of Fractional Differential Equation, North-Holland Mathematics Studies, Elsevier Sciences B.V., Amsterdam, Netherlands, 2006.

[28] T. U. Khan and M. A. Khan, "Generalized conformable fractional operators," Journal of Computational and Applied Mathematics, vol. 346, pp. 378-389, 2019. 